\title{
Electric Propulsion Interactions Code (EPIC): Recent Enhancements and Goals for Future Capabilities
}

\author{
Barbara M. Gardner ${ }^{1}$, Robert A. Kuharski ${ }^{2}$, and Victoria A. Davis ${ }^{3}$ \\ Science Applications International Corporation, \\ 10260 Campus Point Drive, Mail Stop A1, San Diego, California, 92121 \\ and \\ Dale C. Ferguson ${ }^{4}$ \\ NASA Marshall Space Flight Center, Huntsville, Alabama, 35812
}

\begin{abstract}
[Abstract] The Electric Propulsion Interactions Code (EPIC) is the leading interactive computer tool for assessing the effects of electric thruster plumes on spacecraft subsystems. EPIC, developed by SAIC under the sponsorship of the Space Environments and Effects (SEE) Program at the NASA Marshall Space Flight Center, has three primary modules. One is PlumeTool, which calculates plumes of electrostatic thrusters and Hall-effect thrusters by modeling the primary ion beam as well as elastic scattering and charge-exchange of beam ions with thruster-generated neutrals. ObjectToolkit is a 3-D object definition and spacecraft surface modeling tool developed for use with several SEE Program codes. The main EPIC interface integrates the thruster plume into the 3-D geometry of the spacecraft and calculates interactions and effects of the plume with the spacecraft. Effects modeled include erosion of surfaces due to sputtering, re-deposition of sputtered materials, surface heating, torque on the spacecraft, and changes in surface properties due to erosion and deposition. In support of Prometheus I (JIMO), a number of new capabilities and enhancements were made to existing EPIC models. Enhancements to EPIC include adding the ability to scale and view individual plume components, to import a neutral plume associated with a thruster (to model a grid erosion plume, for example), and to calculate the plume from new initial beam conditions. Unfortunately, changes in program direction have left a number of desired enhancements undone. Variable gridding over a surface and resputtering of deposited materials, including multiple bounces and sticking coefficients, would significantly enhance the erosion/deposition model. Other modifications such as improving the heating model and the PlumeTool neutral plume model, enabling timedependent surface interactions, and including EMI and optical effects would enable EPIC to better serve the aerospace engineer and electric propulsion systems integrator. We review EPIC's overall capabilities and recent modifications, and discuss directions for future enhancements.
\end{abstract}

\section{Nomenclature}

NEXIS $=$ ion thruster for use in the Prometheus Project

Prometheus $=$ nuclear-electric propulsion project to reach Jupiter's icy moons

\footnotetext{
${ }^{1}$ Deputy for Operations, Advanced Engineering \& Applied Sciences Division, Advanced Concepts Business Unit, Member AIAA.

${ }^{2}$ Senior Staff Scientist, Advanced Engineering \& Applied Sciences Division, Advanced Concepts Business Unit, Member AIAA.

${ }^{3}$ Senior Staff Scientist, Advanced Engineering \& Applied Sciences Division, Advanced Concepts Business Unit, Member AIAA.

${ }^{4}$ Lead, Constellation Environments Test Integration Group, EM50, AIAA Senior Member.
} 
JIMO = Jupiter Icy Moons Project, later called Prometheus

$S E E \quad=$ Space Environment Effects Program

$N A S C A P-2 K=$ NASA-Air Force Spacecraft Charging Analyzer Program

Hall-effect $=$ transverse force exerted on charge carriers moving in a magnetic field

\section{Introduction}

E LECTRIC propulsion devices typically yield unrivalled specific impulse for spacecraft propulsion. However, electric propulsion devices also inevitably produce induced environments on and around the spacecraft they propel. Components of the plasma plumes generated by electric propulsion come bock onto spacecraft surfaces, and can interact with subsystems, either by contaminating sensitive surfaces, such as optics, solar arrays or thermal control surfaces. In addition, the potential at which the spacecraft floats relative to its surrounding plasma will be changed by the plasma its electric propulsion devices produce. Finally, parasitic currents on spacecraft power systems can be changed by dense electric propulsion plasma plumes. One of the main factors retarding the adoption of electric propulsion systems for spacecraft was concern about the induced environments that electric propulsion brings with it. For this reason, the NASA Space Environment Effects Program (SEE) funded Science Applications International Corporation to develop the Electric Propulsion Interactions Code (EPIC) to enable designers, operators, and users of spacecraft a tool to evaluate the possible interactions and, if necessary, mitigate them. Then, having EPIC allows evaluation of the mitigation techniques employed.

EPIC, a fully interactive, user-friendly computer tool, consists of several modules. The first, PlumeTool, calculates plumes of electrostatic thrusters and Hall-effect thrusters by modeling the primary ion beam as well as elastic scattering and charge-exchange of beam ions with thruster-generated neutrals. The second module, the ObjectToolkit, is a 3-D object definition and spacecraft surface modeling tool developed for use with several SEE Program codes. Finally, the main EPIC interface integrates the thruster plume into the 3-D geometry of the spacecraft and calculates interactions and effects of the plume with the spacecraft. Effects modeled include erosion of surfaces due to sputtering, re-deposition of sputtered materials, surface heating, torque on the spacecraft, and changes in surface properties due to erosion and deposition.

In support of Prometheus I (JIMO), a number of new capabilities and enhancements were made to existing EPIC models. Recent enhancements to EPIC include adding the ability to scale and view individual plume components, to import a neutral plume associated with a thruster (to model a grid erosion plume, for example), and to calculate the plume from new initial beam conditions. This paper will introduce both old familiar users and new prospective users to these new EPIC capabilities.

\section{EPIC Overview}

\section{A. Purpose of EPIC}

The Electric Propulsion Interactions Code (EPIC) is a powerful, flexible, and extensible engineering tool designed to be used to minimize potentially deleterious interactions between electric propulsion effluents and spacecraft systems. Through the use of previously developed code including the MIRIAD (Module Integrator and Rule-based Intelligent Analytic Database) model integrating architecture, and modules developed for Environment WorkBenchl (EWB) and NASCAP-2K, EPIC incorporates models widely used by the space environments community. These include (among others) models of the space plasma environment, orbital motion, sun-pointing of solar arrays, ion engine and Hall-effect thruster effluent plumes, sputtering, and surface contamination by redeposited materials. EPIC employs software already in use by NASA and industry for spacecraft definition, and for thruster and plume modeling, and can be used to perform much-needed trade studies of plume interactions and effects.

EPIC's unique architecture allows new and improved models to be easily incorporated as knowledge and technology advance. Furthermore, EPIC is designed to easily exchange information with other applications. Data exchange between EPIC and NASCAP-2K has been implemented, and further synergism between these two codes is anticipated in the near future. 


\section{B. EPIC}

EPIC is the application with which the user studies interactions and performs trade studies. It is accessed via the EPIC graphical user interface, which is written in the computer-language C\#. Underlying the user interface, the EPIC application consists of MIRIAD utilities and numerous physics modules, written mainly in Fortran 77 with $\mathrm{C}++$ wrappers, and low-level utilities written in C. The Object Toolkit and PlumeTool components of EPIC - which are actually separate applications-may be launched from the EPIC user interface, and the resulting object and plume files can be imported into the EPIC database via the user interface.

\section{Object Toolkit}

Object Toolkit is the standard spacecraft surface definition program for NASA Space Environments and Effects (SEE) Program applications. Originally written for NASCAP-2K, Object Toolkit is extensible to the needs of other applications through an external file. For EPIC, Object Toolkit is extended with the ability to define components (like solar arrays) of a spacecraft and to have them point (at the sun, for example), and with the ability to define electric thrusters, their pointing direction, and their location on the spacecraft. Object Toolkit is written in the Java programming language with the Java3D add-on technology. The output of Object Toolkit is a spacecraft surface definition file written in eXtensible Markup Language (XML) format.

\section{PlumeTool}

PlumeTool is a program for modeling axisymmetric ion engine and Hall-effect thruster plumes. The output of the program is a map of ion densities and velocities, with separate components for the high-energy main beam ions, intermediate-energy elastically scattered ions, and low-energy charge exchange ions. The PlumeTool interface is written in Java with underlying code in Fortran $77, \mathrm{C}++$, and $\mathrm{C}$.

In addition to user-provided boundary conditions, PlumeTool accepts input boundary conditions (thruster output and operating parameters) from an embedded one-dimensional Hall-effect thruster model. In addition to providing the thrust and propellant utilization for plume calculations, spatial and temporal details of this model's results may be copied and imported into a spreadsheet program for further analysis.

\section{E. Main Graphical User Interface}

In Fig. 1 is shown the main graphical user interface for EPIC.

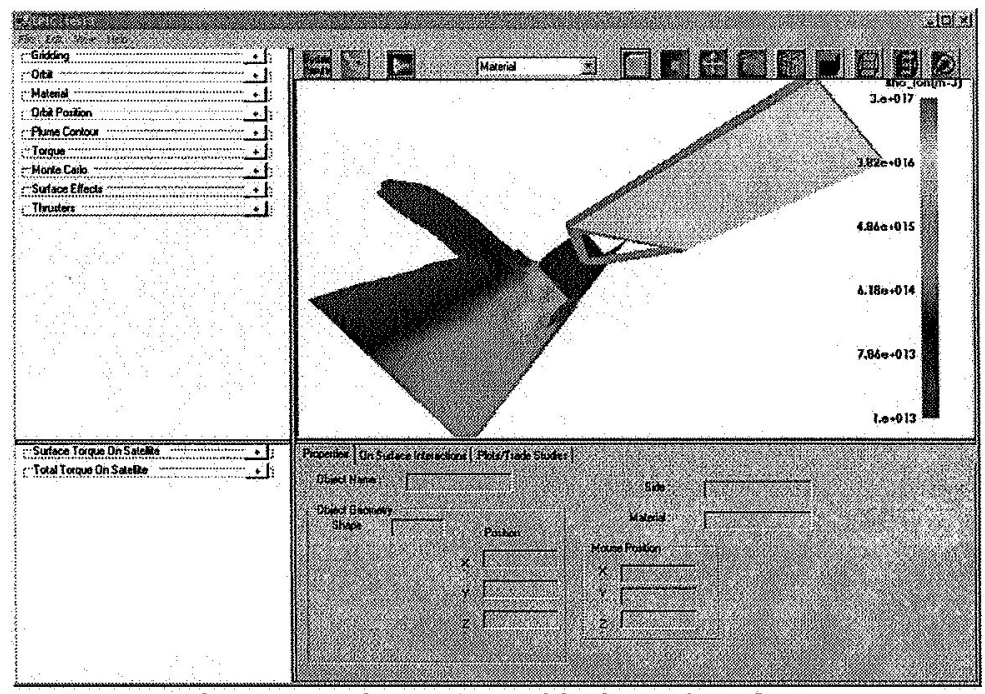

Figure 1. Main EPIC graphical user interface.

The user-friendly aspect of EPIC is clearly shown here, with pull-down menus for interactive input, a three dimensional graphical display, and places for computed and/or specified inputs. The Interaction Input Parameters 
panel is on the top left side of the window. It has several sub-panels, which can be opened to view and edit the different parameter groups. The parameters in this panel are described in Section 5.

The Interaction Output Parameters panel is on the bottom left side of the window. It has two sub-panels, which can be opened to view the calculated values. The parameters in this panel are described in Section 6 .

The 3-D View Display panel is the area in the upper right of the window (showing the spacecraft object and plume particle density contour in Figure 1). The spacecraft and interactions results (in graphical format) are displayed in this panel. The user may also choose to display two-dimensional plasma or neutral plume density contours concurrently with the spacecraft. The three buttons at the top left of this panel are used to update the graphics display with the present values in the database, to launch Object Toolkit, and to launch PlumeTool, respectively. The center-top pull-down menu is used to select the quantity displayed on the spacecraft surfaces. The top-right buttons manipulate the graphics display.

\section{F. Typical Output}

In Fig. 2 is shown an example of a typical output screen from EPIC. This view has the scales for the axes removed, since the work it represents was performed for the Prometheus program (xxx), and most of the design details were considered proprietary and/or EAR/ITAR controlled.

Plume_Ion_Densily
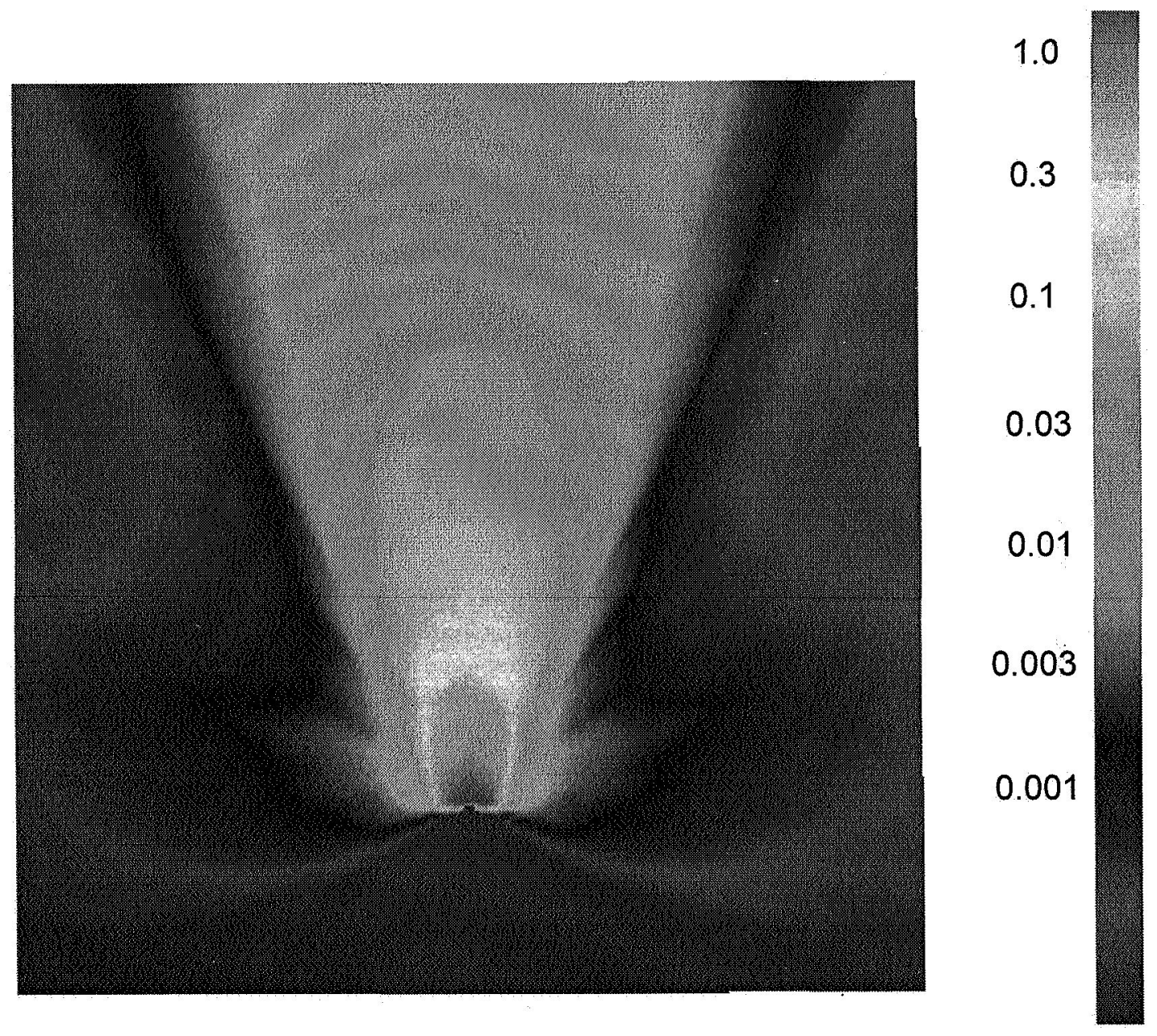

Figure 2. Two-dimensional representation of a NEXIS (Prometheus) thruster plume, calculated by PlumeTool and displayed in EPIC.

Other types of graphical output are also possible, as shown in Fig. 3, also for the NEXIS (Prometheus) thruster. Effects modeled include erosion of surfaces due to sputtering, re-deposition of sputtered materials, surface heating, 
torque on the spacecraft, and changes in surface properties due to erosion and deposition. For a more detailed description of EPIC and its capabilities, see Mikellides et al $^{1}$.

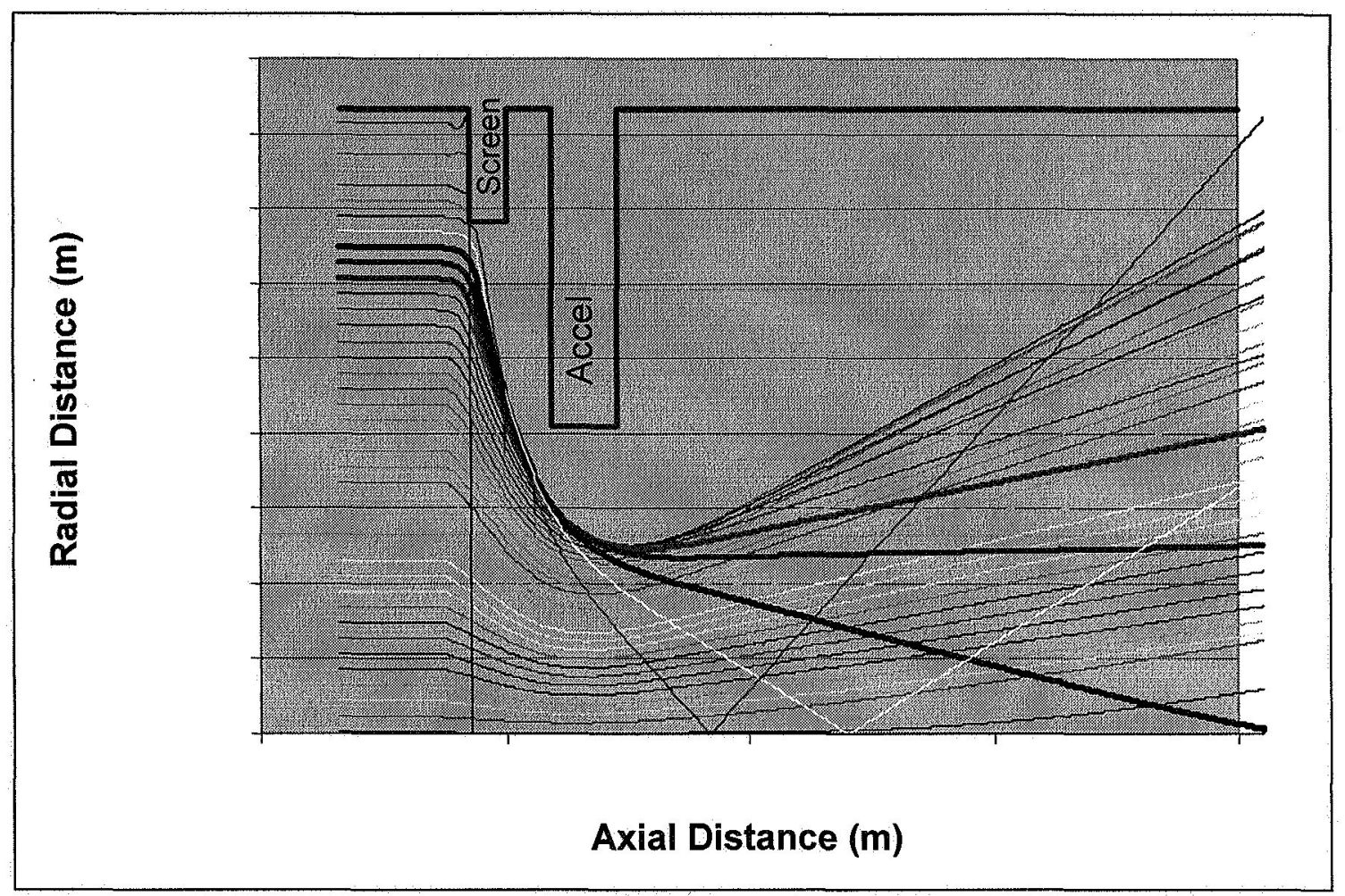

Figure 3. CEX2D (Charge Exchange 2-D)-calculated ion trajectories for a high-current NEXIS beamlet.

\section{Recent EPIC Improvements}

Recent enhancements to EPIC include:

1) adding the ability to scale and view individual plume components,

2) to import a neutral plume associated with a thruster (to model a grid erosion plume, for example),

3) to calculate the plume from new initial beam conditions, and

4) to enable multipie plume capability in NASCAP-2K.

We will discuss these below.

First of all, when considering contamination, especially contamination due to sputtering, it is important to be able to view the individual plume components, since they will cause different amounts of grid-sputtering and sputtering from impacted surfaces. The different plume components now individually considered by EPIC are:

a) Low energy charge-exchange ions. For large, high voltage thrusters, their energy may not be below the sputtering threshold. These can also expand into the backflow region.

b) Intermediate-energy scattered ions. At large angles, these may dominate sputtering, and are clearly seen in the HET thruster data.

c) The high angle portion of the main beam. This extends to about 45 degrees from the beam centerline. The first 15 degrees of beam spread is due to dished grids, and beamlet divergence can cause the beam to spread out to at least 30 degrees.

d) Sputtered grid material (discussed below).

Secondly, a neutral plume capability, for instance from sputtered grid material, was added to EPIC. Now, the contributions of this neutral material from the electric propulsion device to contamination on spacecraft systems can be calculated.

Third, some restrictions on the initial beam conditions in EPIC calculations were lifted, so it can now be used for a more general set of thruster types. Specifically, three carbon-carbon grid material sputtering plumes were added to EPIC capabilities. 
And finally, EPIC was originally coded to handle only one electric propulsion device, since at that time, most spacecraft either built or conceived used only one such device. Now, with electric propulsion being used more and more for station-keeping, and with multiple main thrusters being considered for Prometheus and other large spacecraft, it is important to be able to handle multiple plumes and view their combined interactions with spacecraft systems. For this reason, a multiple plume capability was added to EPIC, and code enhancements were made to allow this capability to be shared with another SEE-funded code, NASCAP-2K, the NASA Air-Force Spacecraft Charging Analysis Program. Now, using EPIC and NASCAP-2K, a spacecraft with multiple simultaneously operating electric propulsion devices can be modeled. NASCAP-2K can now import PlumeTool-formatted plumes under GUI control, calculate potentials in superposed plumes, including surface sheaths, and demonstrate particle tracking in plume potentials. In Fig. 4 is shown a NASCAP-2K plot showing potentials in two superimposed plumes.
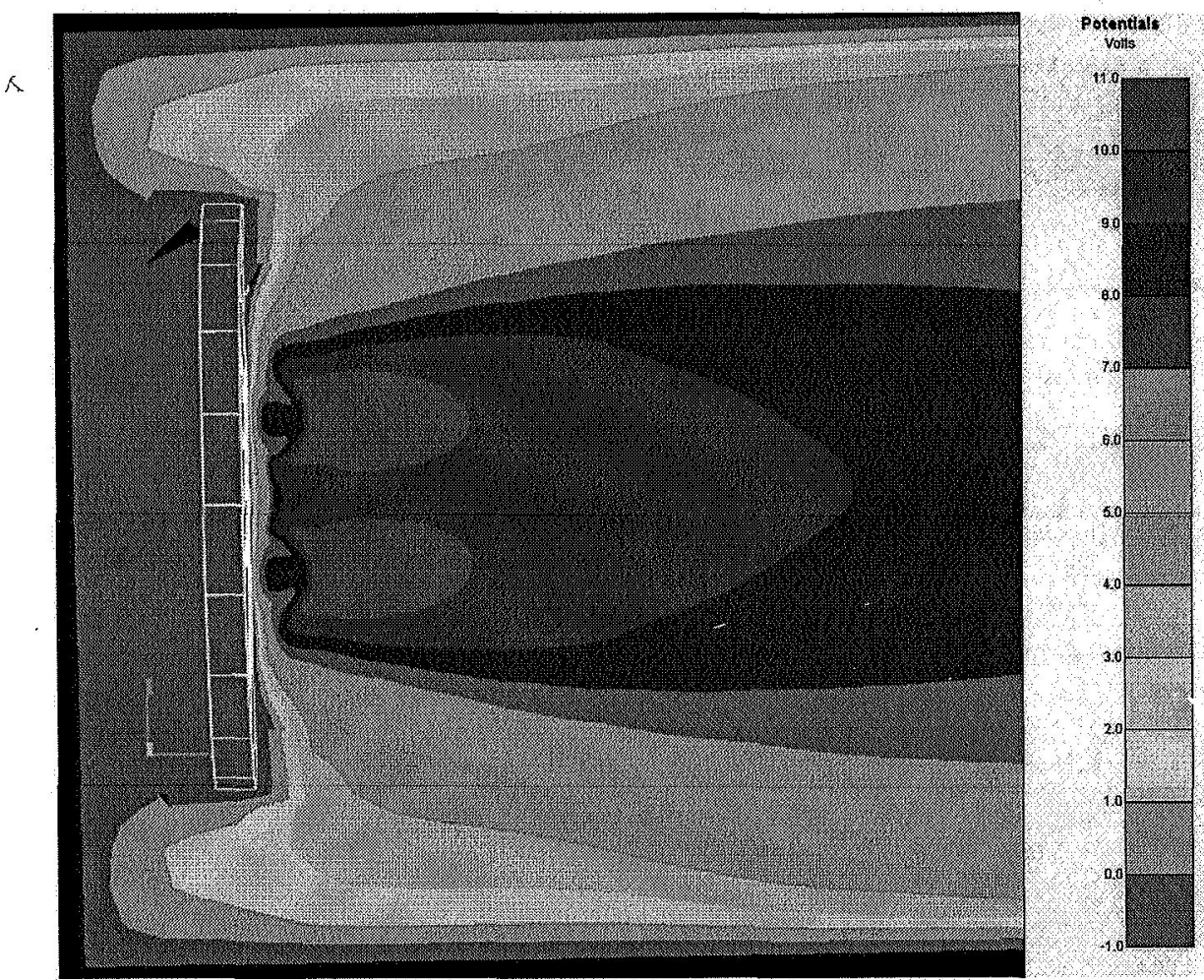

Figure 4. NASCAP-2K calculated self-consistent potentials in two overlapping plumes.

\section{Further Desirable EPIC Improvements}

Because of the demise of the Prometheus Project, and the lack of current funding for the SEE program, many planned improvements to EPIC could not be made. These include variable gridding over a surface and re-sputtering of deposited materials, including multiple bounces and sticking coefficients. These improvements would significantly enhance the erosion/deposition model. Other modifications such as improving the heating model and the PlumeTool neutral plume model, enabling time-dependent surface interactions, and including EMI and optical effects would enable EPIC to better serve the aerospace engineer and electric propulsion systems integrator. However, these improvements must await a sponsor.

Even as it stands, however, EPIC is a valuable tool for predicting, designing for, and operating in the presence of electric propulsion interactions with spacecraft systems.

EPIC and NASCAP-2K are EAR/ITAR controlled items, and may not be shared with non-US citizens, or US citizens in the employ of non-US companies. US citizens may obtain EPIC by filling out the necessary forms at the SEE program web site http://see.msfc.nasa.gov. 


\section{References}

1. Mikellides, I.G., Mandell, M.J., Kuharski, R.A., Davis, V.A., Gardner, B.M., and Minor, J., "The Electric Propulsion Interactions Code," proceedings of the $8^{\text {th }}$ International Spacecraft Charging Technology Conference, Huntsville, Alabama, Oct. 20-24, 2003, NASA/CP-2004-213091. 